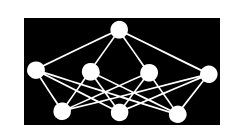

\title{
PRE-CRASH CONTROL STRATEGY OF DRIVER ASSISTANCE SYSTEM
}

\author{
J. Kovanda, V. Rulc,
}

\begin{abstract}
The aim of the article is the optimisation process of the ADAS (Advanced Driver Assistance Systems) control. The methodology is based on the classification of ADAS systems according to the situations of unavoidable accidents. The evaluation of expected consequences uses injury biomechanics, which represents the extended definition of HMI (Human-Machine Interaction). The evaluation of injury mechanism and the machine intervention enables to control this process with the target to minimise the consequent injuries. Then the decision making takes new inputs to the control process and it enriches the multiparametric control of the system with the target to minimise the consequences.
\end{abstract}

Key words: ADAS (Advanced Driver Assistance System), classification, decision making, multiparametric control, HMI (Human-Machine Interaction), BDBSA (Beyond-Design-Basis Safety Assessment)

Received: February 25, 2020

DOI: $10.14311 /$ NNW.2021.31.004

Revised and accepted: February 28, 2021

\section{Introduction}

The importance of car assistance systems continues to grow over time, and it certainly cannot be expected that this should not be any different in the future. On the contrary, it is possible to predict even faster development of these technologies and their transformation from assistance systems to control systems, which will be only assisted by a driver [13].

The complexity of assistance systems makes the situation in assessing their safety relatively complicated. It is not very precise and meaningful if the safety of the subsystem is assessed without taking into account the impact of its behavior on neighboring or superior systems [2]. The evaluation of the level of security of the system is done according to the ability to fulfill the target function $[16,18,15]$. The consequences of the system failure are evaluated in the very limited extension. However, safety analysis of car assistance systems is currently facing both of these problems, and this paper aims to contribute to searching for a solution.

\footnotetext{
*Jan Kovanda - Corresponding author; University of West Bohemia, Faculty of Mechanical Engineering - Regional Technological Institute. Univerzitní 8, 30614 Plzeň, Czech Republic. E-mail: kovanda@rti.zcu.cz

†Vojtěch Rulc; Czech Technical University in Prague, Faculty of Transportation Sciences. Konviktská 20, 11000 Prague 1, Czech Republic.
} 
The paper offers a methodology for optimising a target function of assistance systems regarding the consequences of their potential failures.

The use of the proposed methodology is illustrated in the example of assessing the safety of adaptive cruise control system with the ability of automatic crisis intervention in driving. For that it also uses its own computational models.

A classification methodology being used within this paper is rather classical one. It therefore differs from various modern approaches recently used e.g. in the Neural Network World journal [4, 7, 21, 9, 8, 17].

The conversion to the numerical values of the outputs is required for any optimization methods based on mathematical procedures. The minimization of the traffic accident injuries severity (the finding minimum or maximum of the criterial function is just optimization) cannot work with the verbal classification of injuries (if the fuzzy strategy is not utilised). It is necessary to use a numerical classification expressing the severity of the injury, for example classification of the Abbreviated Injury Scale AIS or appropriate combination of injury criteria [20, 1].

The accident injury classification system design is not easy for the theoretical situation of the driver assistance system failure. The Beyond-Design-Basis Safety Assessment (BDBSA) methodology was designed to manage this most demanding part $[22,23,12,14]$. The scheme of the methodology is depicted in Fig. 1.

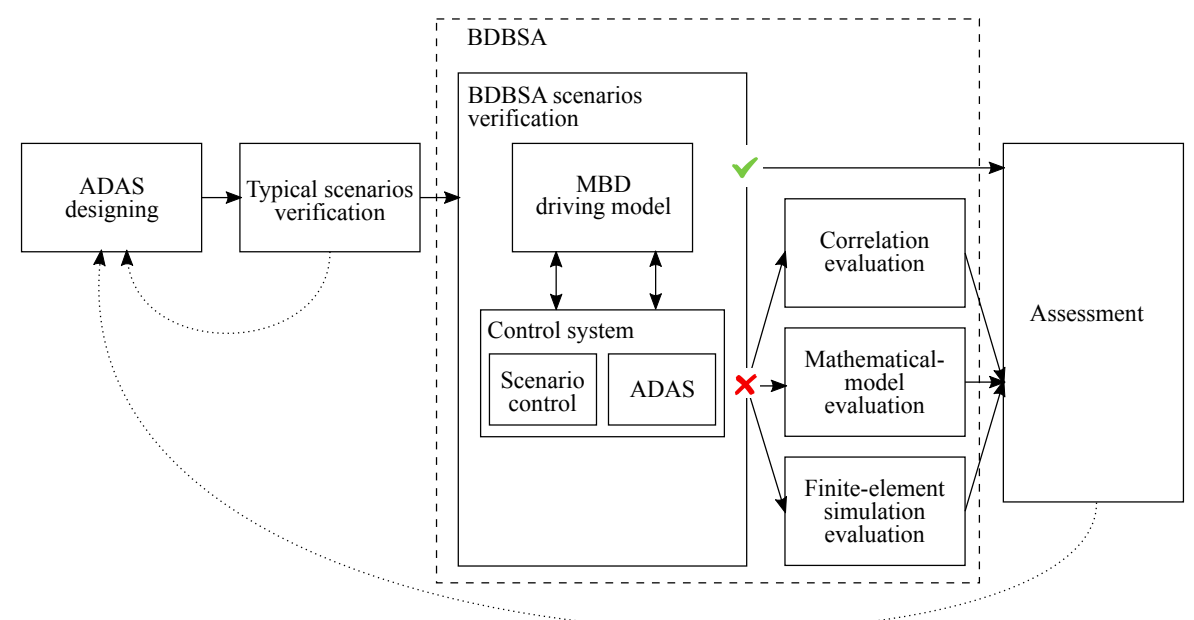

Fig. 1 Decision algorithm methodology [own research]

\section{BDBSA}

Cases beyond the boundary conditions of an accident correspond to situations in which ADAS is unable to prevent an accident. In general, if properly designed, ADAS should be able to avoid impacts in high-probability scenarios and highly 
unlikely scenarios should lead to unavoidable collisions. Due to the very low probability, these cases are not considered at the design stage and are therefore treated as "beyond design cases" (by analogy with the same term in the "defense-in-depth" approach in nuclear engineering [6]). To analyze the impact on passengers and relevant vehicle entities of such cases beyond the design, we use the term BDBSA (beyond-design-basis safety assessment).

The BDBSA corresponds to the third level of the proposed multi-level security approach. As can be expected, not all unavoidable accidents have the same consequences. It is therefore recommended to carry out further analyzes of these conflict scenarios in order to estimate their impact, in particular as regards the injuries of the persons involved. In the following paragraphs, a simple and easy-toimplement approach will be presented as the BDBSA concept, which will analyze the consequences of such unavoidable traffic accidents, when an assistance-driven car collides with a car in front of it. However, the methodology can be easily extended to completely different collision scenarios.

The results of the BDBSA can be used to determine whether the consequences of an unavoidable accident are considered acceptable or not. If they are considered unacceptable, the consequences of the accident should be mitigated by adding additional passive safety systems in the vehicle or by shifting the impact boundary conditions with an additional ADAS safety layer or by modifying the functionality of the system back at the design stage.

\subsection{Safety evaluation}

To evaluate the consequences (with a focus on crew injuries) according to the parameters of the collision (obtained from the previous phase), the presented methodology proposes three possible ways:

1. Correlation evaluation - based on the correlation between impact speed and injuries proceeding for example from data presented in $[19,10]$

2. Mathematical-model evaluation - based on a relatively simple mathematical description of an accident; as an example see $[5,11]$

3. Evaluation by finite-element simulation - based on a detailed simulation of an accident [13]

The first methodology was firstly presented in [12] and is recommended for a simple and fast BDBSA evaluation of predefined specific crash configurations. The other two methodologies of evaluation are then described in [13], and can be used in more general cases than the first methodology. Mainly the evaluation by finite-element simulation.

\section{Optimisation of design process based on FEM simulations}

Finite element modeling (FEM) and simulation (Fig. 2) is the most common way of computer simulations of car crash tests. During the development of passive safety 


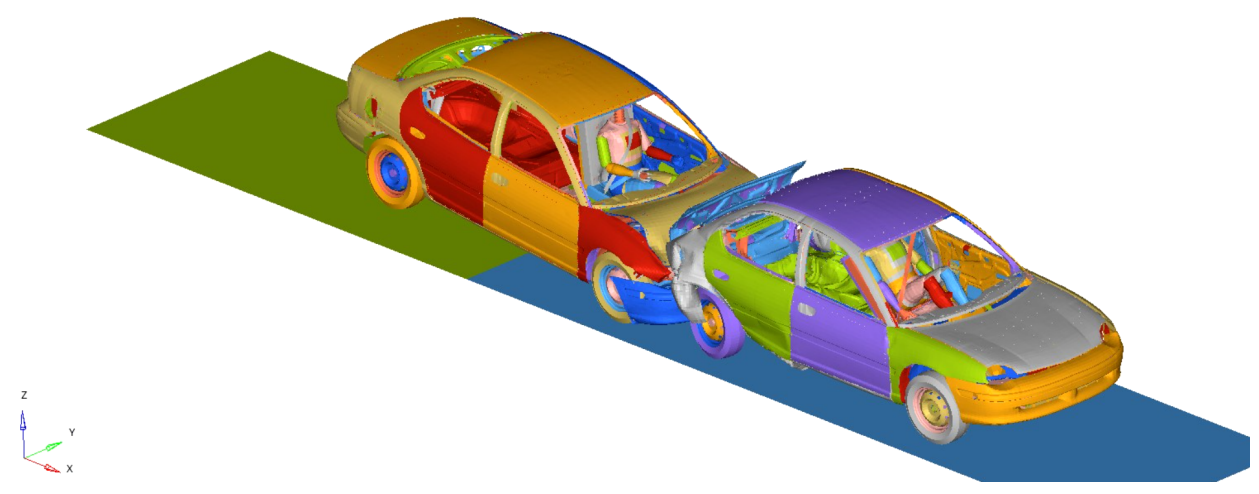

Fig. 2 Crash scenario [own research]

of cars, it enables detailed verification of the functioning of individual elements of passive safety without the need for repeated destructive testing, which is expensive.

This method of modeling is based on assembling models of individual parts of the car, their validation on the basis of a few destructive tests, and finally creating an assembly of all these subsystems into one unit - a complete car and performing virtual tests with it.

Evaluating the consequences of an accident in this way (i.e. FEM) is clearly the most accurate of all the proposed methods, but at the same time the most difficult to process [13]. Both in terms of time resources, computing resources, and in terms of human resources. Creating a sufficiently high-quality finite-element model to simulate the impact of a car so as to reliably simulate the behavior of a real vehicle requires the work of a thoroughly trained expert - a CAE engineer.

If this method is used by car manufacturers, the assembly of such a model should not be a significant obstacle. Automobile manufacturers already routinely build these models to simulate homologation, customer and other crash tests, and could therefore use such a model directly. In the case of using this method, for example by forensic experts, it would mean for them the creation of a whole model from practically zero, which is in practice a disproportionate requirement for their work. They should use one of the procedures described above.

A huge advantage of this approach is its adaptability to any variation of the collision scenario. The high accuracy of the results is ensured with a good computational model for practically any collision configuration.

The decision process based on BDBSA has much higher sensitivity comparing to the direct FEM calculations.

The assumption that BDBSA is necessary for control strategy safety optimization was confirmed in an example of adaptive cruise control system (equipped in green ego car) in scenario, which is depicted in Fig. 3. The scenario assumes a continuous flow of traffic, which suddenly starts to slow down rapidly. The driver of a red vehicle reacts to this situation by quick changing the lane and thus significantly reduces the safety distance of the green vehicle. The traffic flow continues to slow down and the adaptive cruise control system must therefore react. The best way 
Kovanda J., Rulc V.: Pre-crash control strategy of driver assistance system

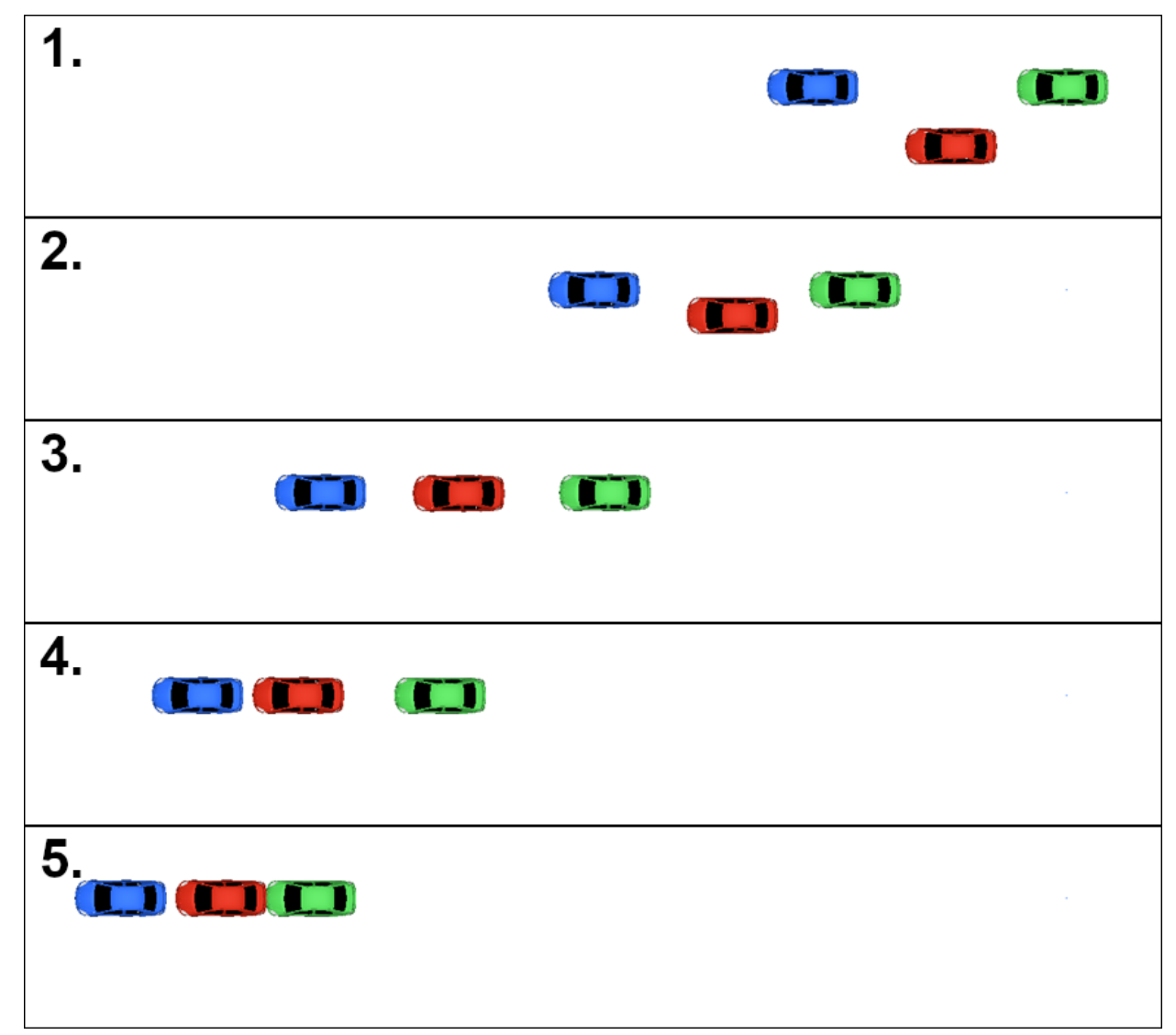

Fig. 3 Pre-crash scenario of strategy heading to the center [own research]

to simulate the parameterized scenarios is using any vehicle dynamic simulation software. Authors used Altair MotionSolve in co-simulation coupling with Altair Activate where the ADAS was modeled.

For the purpose of verifying the assumption, two control strategies of ADAS behavior were proposed in assessing the inevitability of a collision. The first strategy tries to aim the center of the front of the vehicle to the center of the obstacle, the second tries to prevent the collision until the last moment by a combination of braking and evasive maneuver. A detailed description of both strategies is in next sections.

\subsection{Strategy heading to the center}

As soon as the assistance system evaluates that there is an obstacle in the predicted path of the vehicle moving at a higher speed than the set threshold speed relative to the car, it triggers a crisis control strategy. The approach threshold speed is chosen so that the system is not too aggressive and does not overreact to small variations in the speed of the leading vehicle. 
Because we assume an assistance system features, it allows the driver to react as first. The delay for the driver's reaction is based on the current speed and distance from the obstacle (risk of collision) and on the driver's standard reaction time. After this time, the system starts to brake intensively to reduce the impact speed as much as possible.

If an ego vehicle approaches the obstacle at the limit distance and at the same time the radar detects the whole rear part of the car (or the whole obstacle), then it can detect its center and to start the car control. The aim of this procedure is to direct the center of the front part of the ego car to the center of the car in front of it (or the obstacle) to achieve the "full scale" crash.

\subsection{ADAS avoidance strategy}

The second ADAS management strategy has got the same role as the first strategy in the first phase when the obstacle is detected. They differ at a later stage.

If the system determines the expected collision unavoidable, it performs an evasive maneuver by intervening the sine steering movement. Then the ego vehicle moves to the right edge of the road. At the end of this maneuver, it keeps the vehicle in the original direction of travel until the end of the scenario (see Fig. 4).

In this solved unfavorable situation is accepted the assumption, that the width of the roadside is large enough to enable the impact overlap after performing an evasive maneuver $50 \%$ of the width of the vehicle. This type of collision is tested (by even smaller overlap) by both ECE and EuroNCAP tests.

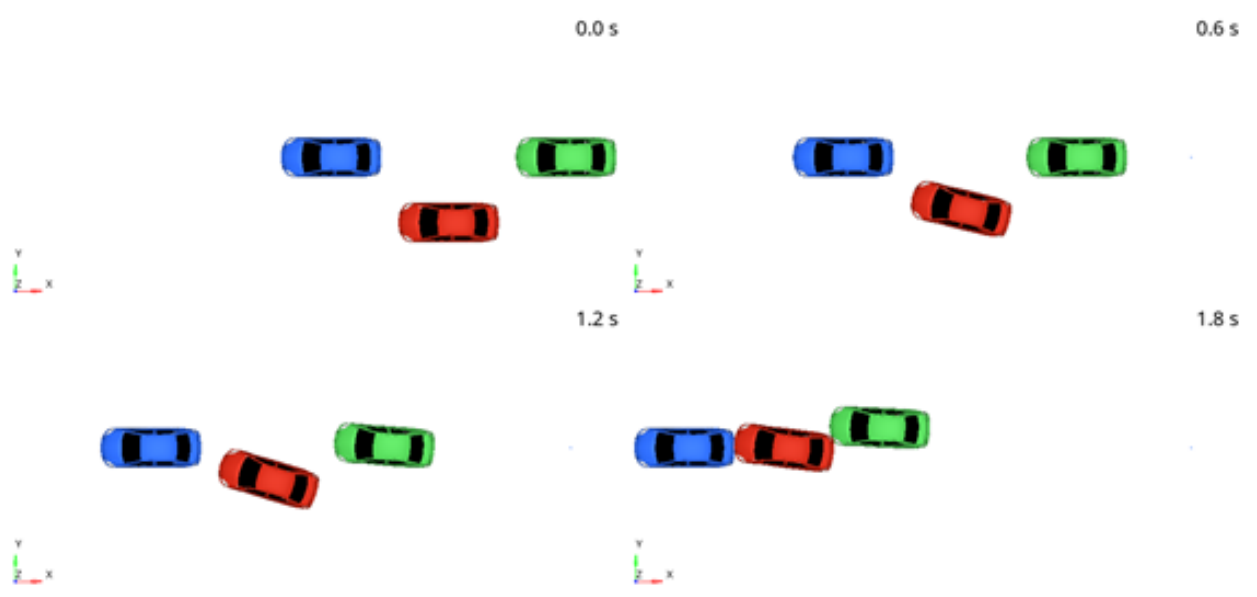

Fig. 4 Sequence of images illustrating the operation of ADAS with an avoidance strategy [own research]

For an initial speed of $80 \mathrm{~km} / \mathrm{h}$, an analysis was made of a comparison of the two considered ADAS control strategies: aiming and avoidance ones. As part of this analysis, 32 scenarios were examined, which, despite the intervention of the assistance system, led to an accident. Of these 9 accident situations had the same input parameters as some of the others. Typically, these were collisions that took 
place in the phase of constant deceleration of the leading (and aggressive) vehicle, when the magnitude of the required final speed has no effect on the parameters of the collision so far. Therefore, 23 finite-element calculations were finally performed.

The results of the analysis are shown in Fig. 5 and Fig. 6 .

\begin{tabular}{|c|c|c|c|c|c|c|c|}
\hline \multirow{2}{*}{\multicolumn{2}{|c|}{$\begin{array}{l}\text { Evasing } \\
80 \mathrm{~km} / \mathrm{h} \\
\text { Collision }\end{array}$}} & \multicolumn{6}{|c|}{$\mathrm{a}_{\text {leader }}\left[\mathrm{ms}^{-2}\right]$} \\
\hline & & 5 & 6 & 7 & 8 & 9 & 10 \\
\hline \multirow{6}{*}{ 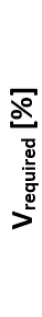 } & 50 & 0 & 0 & 0 & 0 & 0 & 0 \\
\hline & 40 & 0 & 0 & 0 & 0 & 0 & 0 \\
\hline & 30 & 0 & 0 & 0 & 0 & 1 & 1 \\
\hline & 20 & 0 & 0 & 1 & 1 & 1 & 1 \\
\hline & 10 & 0 & 1 & 1 & 1 & 1 & 1 \\
\hline & 0 & 0 & 1 & 1 & 1 & 1 & 1 \\
\hline
\end{tabular}

\begin{tabular}{|c|c|c|c|c|c|c|c|}
\hline \multirow{2}{*}{\multicolumn{2}{|c|}{$\begin{array}{l}\text { Aiming } \\
80 \mathrm{~km} / \mathrm{h} \\
\text { Collision }\end{array}$}} & \multicolumn{6}{|c|}{$a_{\text {leader }}\left[\mathrm{ms}^{-2}\right]$} \\
\hline & & 5 & 6 & 7 & 8 & 9 & 10 \\
\hline \multirow{6}{*}{ 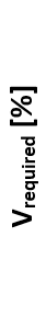 } & 50 & 0 & 0 & 0 & 0 & 0 & 0 \\
\hline & 40 & 0 & 0 & 0 & 0 & 0 & 0 \\
\hline & 30 & 0 & 0 & 0 & 0 & 1 & 1 \\
\hline & 20 & 0 & 0 & 1 & 1 & 1 & 1 \\
\hline & 10 & 0 & 1 & 1 & 1 & 1 & 1 \\
\hline & 0 & 0 & 1 & 1 & 1 & 1 & 1 \\
\hline
\end{tabular}

Fig. 5 Comparison of both strategies in terms of whether the strategy leads to collision [own research]

\begin{tabular}{|c|c|c|c|c|c|c|c|}
\hline \multirow{2}{*}{\multicolumn{2}{|c|}{$\begin{array}{c}\text { Evasing } \\
80 \mathrm{~km} / \mathrm{h} \\
\text { HIC }\end{array}$}} & \multicolumn{6}{|c|}{$\mathrm{a}_{\text {leader }}\left[\mathrm{ms}^{-2}\right]$} \\
\hline & & 5 & 6 & 7 & 8 & 9 & 10 \\
\hline \multirow{6}{*}{ 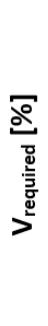 } & 50 & 0 & 0 & 0 & 0 & 0 & 0 \\
\hline & 40 & 0 & 0 & 0 & 0 & 0 & 0 \\
\hline & 30 & 0 & 0 & 0 & 0 & 12 & 18 \\
\hline & 20 & 0 & 0 & 9 & 42 & 57 & 69 \\
\hline & 10 & 0 & 12 & 15 & 30 & 57 & 69 \\
\hline & 0 & 0 & 27 & 15 & 30 & 57 & 69 \\
\hline
\end{tabular}

\begin{tabular}{|c|c|c|c|c|c|c|c|}
\hline \multirow{2}{*}{\multicolumn{2}{|c|}{$\begin{array}{c}\text { Aiming } \\
80 \mathrm{~km} / \mathrm{h} \\
\text { HIC }\end{array}$}} & \multicolumn{6}{|c|}{$a_{\text {leader }}\left[\mathrm{ms}^{-2}\right]$} \\
\hline & & 5 & 6 & 7 & 8 & 9 & 10 \\
\hline \multirow{6}{*}{ 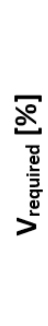 } & 50 & 0 & 0 & 0 & 0 & 0 & 0 \\
\hline & 40 & 0 & 0 & 0 & 0 & 0 & 0 \\
\hline & 30 & 0 & 0 & 0 & 0 & 47 & 62 \\
\hline & 20 & 0 & 0 & 38 & 64 & 89 & 107 \\
\hline & 10 & 0 & 26 & 60 & 74 & 92 & 107 \\
\hline & 0 & 0 & 44 & 60 & 74 & 92 & 107 \\
\hline
\end{tabular}

Fig. 6 Comparison of both strategies with BDBSA methodology [own research]

The chart in Fig. 5 provides the results of simulation of a given scenario with respect to the existence of the collision $(1 \ldots$ crash, $0 \ldots$ no crash). The chart in Fig. 6 presents the expected HIC or HPC (Head Injury (Performance) Criterion) in the case of collision. These data are the background for the vehicle assistance system decision making and the algorithm optimisation.

A comparison of the two strategies refutes the original assumption that the avoidance strategy will have a negative effect on the safety of the crew. In a deliberately unfavorable scenario for this strategy, this steering strategy always led to a front-rear overhang. This impact configuration involves only part of the deformation zones in reducing the consequences of the accident, and therefore the consequences of the accident should be more severe according to the original assumption. 
However, this turned out not to be the case. This can be caused by several factors:

- The flexibility of deformation zones of the modeled car (and cars in general) is optimized to achieve the best results in standardized impact tests, which, however, take place under more unfavorable conditions than achieved in simulated scenarios (higher impact speed, stationary barrier, deformable barrier less than the collision partner, or not at all, etc.). This can then lead to deformation zones being too stiff for impacts at speeds, which for a given structure of the deformation zone, are lower than the critical deformation rate of the beam. Therefore, if fewer of them are involved in the deformation process, it can have a positive effect on the resulting accelerations acting on the crew.

- In an impact with an overlap, collision partners spin around each other, which has an effect on the dissipation of kinetic energy.

- The measured value of HPC takes into account only the sliding components of the acceleration acting on the head. Unfortunately, it neglects the rotational components, which, especially in the collision event where rotations occur, can affect the accuracy of the result to some extent, although probably in this case only to a small extent.

The comparison also shows the benefit of this methodology compared to the current state of safety testing of assistance systems. It can be seen that both management strategies lead to the same number of accidents, and even accidents occur with the same collision scenario parameters. Using the methodologies used so far, both strategies would be evaluated as of the same quality. However, the presented methodology shows that from the point of view of security, there is a significant difference between the two strategies (Fig. 6).

The conclusion of the comparison may be a recommendation to verify the avoidance strategy on a wider range of collision scenarios, because the analysis speaks significantly in favor of this solution.

Of course, it would be possible to use this methodology to identify situations where which strategy is more advantageous, respectively safer, and which ADAS should choose in which situation.

\section{Optimisation process}

For optimization of safety behaviour of ADAS, i.e. minimization of hazard intensity, a following optimization approach, based on the multiparametric control of the system [3], can be used:

Function $f(x)$ to be minimized by iterations:

$$
f_{i}(x) \leq \sup _{i}(x) ; i=1,2, \ldots, n .
$$

After linear decomposition:

$$
f_{\xi}(a x+b y+c z+\ldots) \leq a \cdot f_{\xi}(x)+b \cdot f_{\xi}(y)+c \cdot f_{\xi}(z)+\ldots
$$




\section{Kovanda J., Rulc V.: Pre-crash control strategy of driver assistance system}

I.e. vehicle parameters:

$v_{x} \ldots$ longitudinal velocity

$v_{y}$... lateral velocity

$a_{x} \ldots$ longitudinal acceleration

$a_{x}$... lateral acceleration

Decision which are given by ADAS:

$r_{1} \ldots$ brake

$r_{2} \ldots$ accelerate

$r_{3} \ldots$ turn left

$r_{4} \ldots$ turn right

Then finding a local minimum should be proven on specific cost function:

$$
g(x)=a \cdot H P C+b \cdot T T I+c \cdot 3 M S+d \cdot V C+e \cdot N I C+\ldots,
$$

where $H P C, T T I, V C, N I C, 3 M S$ and many other biomechanical criteria. Presented are some examples of the criteria, the complex information including formulas are published in [1].

HPC is the Head Performance Criterion, frequently used for head injury evaluation based on measured linear acceleration in the head gravity centre of the crash test dummy. The definition is:

$$
H P C=\left\{\left(t_{2}-t_{1}\right)\left[\frac{1}{\left(t_{2}-t_{1}\right)} \int_{t_{2}}^{t_{2}} a(t) d t\right]^{2,5}\right\}_{\max },
$$

where $t_{1}$ is start of interval, $t_{2}$ end of interval and $a(t)$ acceleration in time.

TTI is the Thoracic Trauma Index, the criterion takes into evaluation the lateral acceleration measured on the ribs and 12th thoracic vertebrae.

$$
T T I=0,5 \cdot(R I B Y+T 12 Y),
$$

where:

$R I B Y$ maximum acceleration detected on the upper and lower rib $[\mathrm{g}]$ $T 12 Y$ maximum acceleration of the lower spine (vertebrae Th12) $[\mathrm{g}]$

$\mathrm{VC}$ is a Viscous Criterion preventing the development and propagation of viscous wave through the internal organs on the base of measurements of thorax deformation and the speed of this deformation.

$$
V C=\frac{d[D(t)]}{d t} \cdot \frac{D(t)}{D}
$$

where:

$D(t)$ deformation of chest (in time)

$D \quad$ Defined constant - usually half of dimension of chest $[\mathrm{m}]$

NIC is the Neck Injury Criterion based on the forces and torque measurements on the cervical spine.

3MS is a very general injury criterion evaluation acceleration signal and its remaining at the signal level in the cumulative time $3 \mathrm{~ms}$. 
There are many other useful injury criteria suitable for the evaluation process.

The combination of suitable criterions provides the estimation of expected suffered polytrauma during the crash.

The injury biomechanics enables to create the chart if expected consequences for various crash scenarios. On this base the multiparametric control of ADAS is a tool for minimising the severity in injuries during unavoidable accidents.

Utilising this approach the HMI gets a new dimension - the interaction between the human body and the machine in the case of an accident. The interaction is done by the contact forces, acceleration fields, etc., and the proper design and the restraint systems help to control the injury mechanism.

\section{Conclusion}

The target of the study is the classification of Advanced Driver Assistance Systems (ADAS) beyond the basic design bases, i.e. BDBSA method (Beyond-Design-Basis Safety Assessment). Taking into consideration extended HMI (Human-Machine Interaction) by the injury biomechanics, the decision making is based on the consequences of the unavoidable accidents. The multiparametric control enables to include the injury assessment into the target function. The decision process covers the entire process of precrash vehicle dynamics and the crash mechanics.

\section{Acknowledgement}

This research has been realized using the support of Technological Agency, Czech Republic, programme National Competence Centres, project \# TN01000026 Josef Bozek National Center of Competence for Surface Transport Vehicles.

This support is gratefully acknowledged.

\section{References}

[1] BUNDESANSTALT FÜR STRASSENWESEN. Crash analysis criteria description. 2008. Available also from: http://mdvfs.org/download/crash-analysiscriteria-2-1-1/.

[2] COlOmbaroni C., FUSCO G. Artificial Neural Network Models for Car Following: Experimental Analysis and Calibration Issues. Journal of Intelligent Transportation Systems. 2014, 18, pp. 5-16, doi: 10 . $1080 / 15472450$. 2013 . 801717. ISSN 1547-2450.

[3] ELNOUR E. Autonomous Driving Tactical decisions \& Execution for overtaking maneuver. 2020.

[4] GULZAT T., LYAZAT N., SILADI V., GULBAKYT S., MAKSATBEK S. Research on predictive model based on classification with parameters of optimization. Neural Network World. 2020, 30, doi: 10.14311/NNW.2020.30.020. ISSN 23364335.

[5] IONUT R.A., CORNELIU C., BOGDAN T. Mathematical model validated by a crash test for studying the occupant's kinematics and dynamics in a cars' frontal collision. International Journal of Automotive Technology. 2017, 18, pp. 1017-1025, doi: 10.1007/s12239-017-0099-0. ISSN 1229-9138. 
Kovanda J., Rulc V.: Pre-crash control strategy of driver assistance system

[6] LACHAUME J.-L., MILLER D., RZENTKOWSKI G., LAHTINEN N., VALTONEN K., FOUCHER L., HARIKUMAR S.S., YAMADA T., SHARAFUTDINOV R., KUZNETSOV M. Implementation of defence in depth at nuclear power plants. lessons learnt from the fukushima daiichi accident. Nuclear Energy Agency, Organisation for Economic Co-Operation and Development, Tech. Rep. 2016.

[7] LI W., LI B., GUO H., FANG Y., QIAO F., ZHOU S. THE ECG SIGNAL CLASSIFICATION BASED ON ENSEMBLE LEARNING OF PSO-ELM ALGORITHM. Neural Network World. 2020, 30, doi: 10.14311/NNW.2020.30.018. ISSN 23364335.

[8] MILOŠEVIĆ N., RACKOVIĆ M. CLASSIFICATION BASED ON MISSING FEATURES IN DEEP CONVOLUTIONAL NEURAL NETWORKS. Neural Network World. 2019, 29, doi: 10.14311/NNW.2019.29.015. ISSN 12100552.

[9] MOOS P., NOVÁK M., VOTRUBA Z. Parametric sensitivity in decision making process. Neural Network World. 2020, 30, doi: 10.14311/NNW . 2020.30.003. ISSN 23364335.

[10] OTTE D. Signifikanz von HWS-Distorsionen bei Strassenverkehrsunfällen aus der Sicht der Erhebungen am Unfallort GIDAS. Whiplash 2010. 2010.

[11] PAWLUS W., KARIMI H.R., ROBBERSMYR K.G. Mathematical modeling of a vehicle crash test based on elasto-plastic unloading scenarios of spring-mass models. The International Journal of Advanced Manufacturing Technology. 2011, 55, pp. 369-378, doi: 10.1007/s00170-010-3056-x. ISSN 0268-3768.

[12] REITERER F., ZHOU J., KOVANDA J., RULC V., KEMKA V., del RE L. BeyondDesign-Basis Evaluation of Advanced Driver Assistance Systems. In: Beyond-DesignBasis Evaluation of Advanced Driver Assistance Systems, 2019, pp. 2119-2124. Available also from: https://ieeexplore.ieee.org/document/8813893/, doi: 10 . 1109/IVS. 2019.8813893. ISBN 978-1-7281-0560-4.

[13] RULC V. Vehicle Safety and Assistance Systems. 2020. PhD thesis.

[14] RULC V., KOVANDA J., RE L.D., KEMKA V., REITERER F., ZHOU J. Advanced Driver Assistance Systems - Analysis of Collision Scenarios. 2019. ISBN 978-80-2610890-0.

[15] SCHÄDLER O., MÜLLER S., GRÜNDL M. Experimental evaluation of the controllability of interacting advanced driver assistance systems. 2016. Available also from: http://www.crcnetbase.com/doi/10.1201/b21185-34. ISBN 9781138028852.

[16] SCHULDT F., SAUST F., LICHTE B., MAURER M., SCHOLZ S. Effiziente systematische Testgenerierung für Fahrerassistenzsysteme in virtuellen Umgebungen. AAET2013 - Automatisierungssysteme, Assistenzsysteme Und Eingebettete Systeme Für Transportmittel. 2013.

[17] SHARIF S.M.A., MAHBOOB M. DEEP HOG: A HYBRID MODEL TO CLASSIFY BANGLA ISOLATED ALPHA-NUMERICAL SYMBOLS. Neural Network World. 2019, 29, doi: 10.14311/NNW.2019.29.009. ISSN 12100552.

[18] SHOUKRY Y., TABUADA P., TSUEI S., MILAM M.B., GRIZZLE J.W., AMES A.D. Closed-form controlled invariant sets for pedestrian avoidance. In: Closed-form controlled invariant sets for pedestrian avoidance, 2017, pp. 1622-1628. Available also from: http://ieeexplore.ieee.org/document/7963185/, doi: 10.23919/ACC. 2017.7963185. ISBN 978-1-5090-5992-8.

[19] STIGSON H., KULLGREN A., ROSÉN E. Injury risk functions in frontal impacts using data from crash pulse recorders. 2012. Available also from: https://www . ncbi.nlm.nih.gov/pmc/articles/PMC3503411/. 
[20] WiSMAns J., JANSSEN E., BEUSEnBERG M., KOPPENS W., HAPPEE R., BOVENDEERD P. Injury Biomechanics (4J610). 2000.

[21] WU H., SONG Q., JIN G. Underwater acoustic signal analysis: preprocessing and classification by deep learning. Neural Network World. 2020, 30, doi: 10.14311/ NNW. 2020.30.007. ISSN 23364335.

[22] ZHOU J., RE L.D. Identification of critical cases of ADAS safety by FOT based parameterization of a catalogue. In: Identification of critical cases of ADAS safety by FOT based parameterization of a catalogue, 2018, doi: 10.1109/ASCC. 2017.8287212. ISBN 9781509015733.

[23] ZHOU J., RE L.D. Safety Verification of ADAS by Collision-free Boundary Searching of A Parameterized Catalog. In: Safety Verification of ADAS by Collision-free Boundary Searching of A Parameterized Catalog, 2018, doi: 10.23919/ACC. 2018. 8431291. ISBN 9781538654286. 